\title{
Use of an Isotope-Coded Mass Tag (ICMT) Method To Determine the Orientation of Cholesterol Oxidase on Model Membranes
}

\author{
John E. Gadbery ${ }^{\dagger}$ and Nicole S. Sampson*, ${ }^{\dagger},+0$ \\ ${ }^{\dagger}$ Biochemistry and Structural Biology Graduate Program, Stony Brook University, Stony Brook, New York 11794-5215, United \\ States \\ ${ }^{\ddagger}$ Department of Chemistry, Stony Brook University, Stony Brook, New York 11794-3400, United States
}

Supporting Information

ABSTRACT: Although the interfacial membrane protein cholesterol oxidase is structurally and kinetically well-characterized, its orientation in and mode of interaction with cholesterol-containing membranes have not been established. Cholesterol oxidase can alter the structure of the cell membrane in pathogenic bacteria and is thus a potential antimicrobial drug target. We recently developed a mass spectrometry-based isotope-coded mass tag (ICMT) labeling method to monitor the real-time solvent-accessible surface of peripheral membrane proteins, such as cholesterol oxidase. The ICMT strategy utilizes maleimide-based isotope tags that covalently react with cysteine residues. In this study, by comparing the ICMT labeling rates of cysteine variants of cholesterol oxidase, we

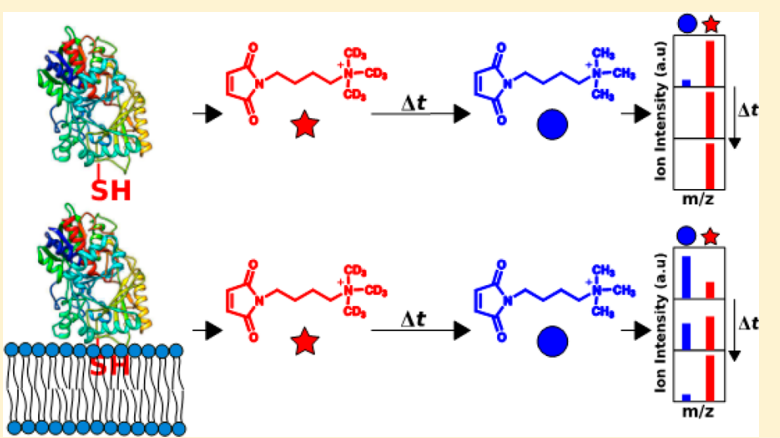
determined which residues of the protein were engaged with the protein-lipid interface. We found that upon addition of cholesterol-containing lipid vesicles, four cysteine residues in a cluster near the substrate entrance channel are labeled more slowly with ICMT probes than in the absence of vesicles, indicating that these four residues were in contact with the membrane surface. From these data, we generated a model of how cholesterol oxidase is oriented when bound to the membrane. In conclusion, this straightforward method, which requires only microgram quantities of protein, offers several advantages over existing methods for the investigation of interfacial membrane proteins and can be applied to a number of different systems.

$\mathrm{C}$ holesterol oxidase (ChoA, UniProtKB entry P12676) is a $55 \mathrm{kDa}$ peripheral membrane protein that transiently associates with the cell membrane to abstract cholesterol and convert it to cholest-4-en-3-one. ${ }^{1-3}$ In addition to its use as an analytical tool to measure serum cholesterol levels, ChoA is produced by a wide variety of pathogenic bacteria, such as Rhodococcus equi. ChoA facilitates the lysis of the cell membrane during $R$. equi infection and may act synergistically with antioxidative enzymes, as well, promoting intracellular survival of the bacteria. Importantly, there are no human homologues to ChoA, making ChoA an attractive antimicrobial target. ${ }^{2,4}$ ChoA is a well-studied protein, and several highresolution crystal structures have been determined, detailing its structure. $^{5,6}$ Additional crystal structures, as well as biochemical studies, have elucidated the mechanism by which ChoA catalyzes the oxidation and isomerization of cholesterol. ${ }^{7-12}$ The structure of ChoA suggests that conformational changes must occur to allow cholesterol access to the deeply buried active site.

Despite this wealth of knowledge about the structure and function of ChoA, there is no direct evidence showing how ChoA is oriented at the membrane interface or whether conformational changes do in fact occur. To address this gap in knowledge, we have used our previously reported ${ }^{13}$ isotopecoded mass tag (ICMT) labeling method to probe cysteine residues in ChoA. On the basis of the relative accessibilities of these residues in the presence and absence of lipid vesicles, we can determine which residues interact at the membrane interface.

Because membrane-protein complexes are dynamic and unstable, traditional methods of structure determination such as X-ray crystallography and nuclear magnetic resonance are challenging. Alternative methods for studying protein dynamics at the membrane interface, including Förster resonance energy transfer, ${ }^{14-16}$ electron paramagnetic resonance spectroscopy, ${ }^{17-20}$ and fluorescence quenching spectroscopy, ${ }^{21,22}$ can yield valuable information. However, these methods require the attachment of probes that often contain long linkers that separate the reporting group from the local environment in which the probes are bound. Additionally, these methods typically can monitor only one site at a time, requiring the preparation of several proteins with a single modification. The ICMT method can overcome many of these limitations, and in this work, we demonstrate that it can be used to assess the interfacial structure of ChoA.

Received: July 24, 2018

Revised: August 15, 2018

Published: August 20, 2018 
(a)

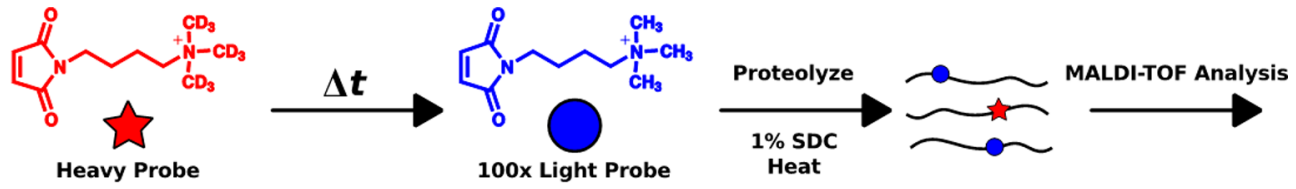

(b)

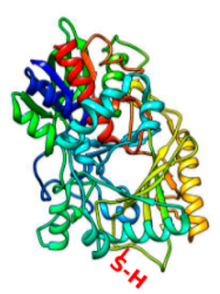

(e)

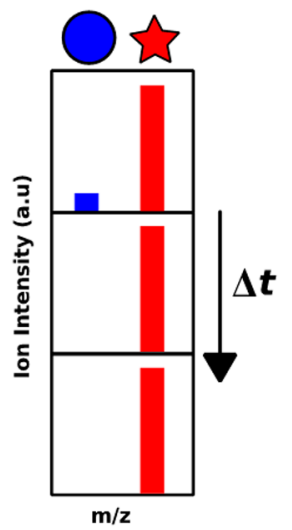

Exposed

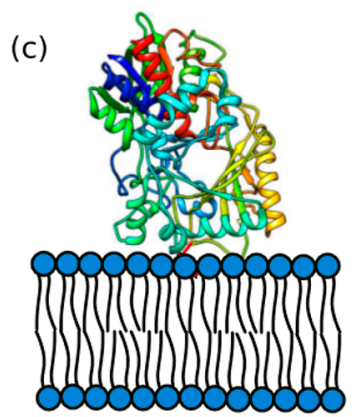

(f)

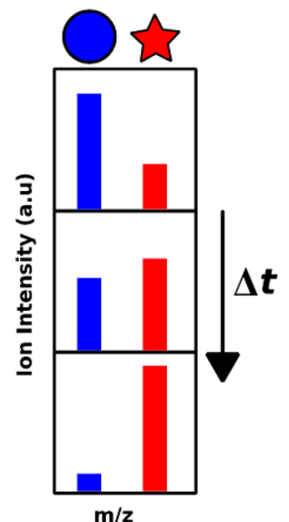

Dynamic (d)

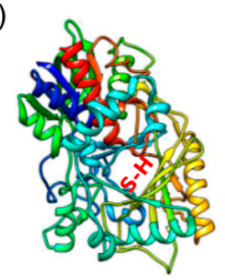

(g)

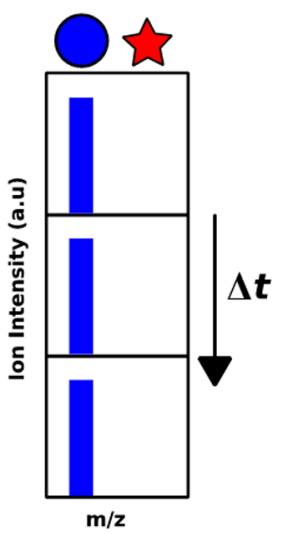

Protected

Figure 1. Two-step ICMT labeling procedure. (a) Scheme of the ICMT labeling procedure. Cysteine-containing ChoA (Protein Data Bank entry $1 \mathrm{MXT}$ ) is labeled with the (red) heavy probe (c) in the presence of vesicles or (b and d) in buffer alone. At specified time points, a 100-fold molar excess of (blue) light probe is added; then the membrane is solubilized, and the protein is unfolded by being heated in the presence of a detergent. The unfolded protein is digested into short peptides, which are analyzed via MALDI-TOF MS. The position of a Cys residue with respect to the membrane can easily be determined from the MALDI-TOF spectra: ( $b$ and e) exposed residue in buffer alone, ( $c$ and $f$ ) the same residue at the protein-lipid interface upon addition of vesicles, and ( $\mathrm{d}$ and $\mathrm{g}$ ) a solvent-protected residue.

The membrane-impermeable ICMT probes, synthesized in both light and heavy (deuterated) forms, consist of a thiolreactive maleimide group linked to a positively charged quaternary ammonium group that contains either methyl groups (light probe) or deuteromethyl groups (heavy probe) (Figure 1). At physiological $\mathrm{pH}$, maleimides readily react with solvent-accessible cysteines to form a covalent thioether linkage, ${ }^{23}$ whereas they react more slowly with thiols in hydrophobic environments. ${ }^{24}$ In an aqueous environment, the $\mathrm{p} K_{\mathrm{a}}$ of a sulfhydryl is between 8 and 9 , and there is sufficient concentration of the reactive thiolate anion for facile labeling. In a hydrophobic environment, the $\mathrm{p} K_{\mathrm{a}}$ is increased to nearly 14 , making a thiol buried in the membrane far less reactive. ${ }^{23}$ Using transmembrane helices, we showed previously that thiols buried to a depth of $\sim 7 \AA$ in lipid vesicles react with ICMT probes at rates as slow as $1 / 8$ th of those of exposed thiols. Thiols buried at the center of the membrane do not react until the membrane is lysed. ${ }^{13}$

The ICMT method is analogous to earlier cysteine-scanning methods for mapping the structures of membrane proteins. ${ }^{25,26}$ In these methods, amino acids are substituted one at a time with cysteine and labeled with a thiol-reactive probe. The boundary between exposed and membrane-embedded regions of the protein can be determined by comparing the accessibility of each cysteine to the probe. The ICMT method consists of a two-step labeling procedure (Figure 1). First, the native protein is labeled with the heavy probe. In the second labeling step, an excess of the light probe is added at specified time points. Then the protein is unfolded, and the membrane is solubilized by being heated in the presence of a detergent to ensure complete labeling of the thiol by the light probe. Once labeling is complete, the protein is proteolyzed, and the resulting labeled peptides are analyzed by matrix-assisted laser desorption ionization time-of-flight mass spectrometry (MALDI-TOF MS). The accessibility of the targeted cysteine determines the ratio of labeling by the heavy probe relative to that by the light probe. Accessibility is a function of the residue's position in the protein and its position relative to the membrane interface. Comparison of the labeling ratios in the presence and absence of membranes provides information about the location of a specific cysteine with respect to the membrane interface.

In this work, we used the ICMT method described above to determine the orientation of ChoA bound to cholesterolcontaining vesicles. Our results confirm that this method is a useful tool for determination of the interfacial structure of proteins. 


\section{MATERIALS AND METHODS}

Materials. Sodium dodecyl sulfate, tryptone, HEPES, ampicillin, sodium dihydrogen phosphate, sodium hydrogen phosphate, and TCEP were purchased from Fisher Scientific (Hampton, NH). Triton X-100, sodium deoxycholate, cholesterol, and bovine serum albumin were purchased from Sigma-Aldrich (St. Louis, MO). 2,5-Dihydroxybenzoic acid was purchased from TCI (Portland, OR). Primers for polymerase chain reaction (PCR) mutagenesis and DNA sequencing were purchased from Eurofins MWG Operon (Louisville, KY). Restriction enzymes and trypsin were purchased from New England Biolabs (Ipswich, MA). Chymotrypsin was purchased from Roche Applied Science (Indianapolis, IN). A QuikChange Site-Directed Mutagenesis Kit was purchased from Agilent (Santa Clara, CA). A Plasmid Mini-Prep Purification Kit was purchased from Qiagen (Germantown, MD). Isopropyl $\beta$-D-1-thiogalactopyranoside was purchased from Denville Scientific (Holliston, MA). Phospholipids were purchased from Avanti Polar Lipids (Alabaster, AL).

General Methods. DNA was sequenced either at the Stony Brook University Sequencing Facility with an Applied Biosystems 3730 DNA analyzer or by Genewiz with an Applied Biosystems 3730xl DNA analyzer. Cells were disrupted with a Constant Systems (Daventry, U.K.) cell disrupter. As a liquid microbial growth medium, we used $2 \times Y T$ medium (16 g of tryptone, $10 \mathrm{~g}$ of yeast extract, and $5 \mathrm{~g}$ of sodium chloride per liter). An MTP384 ground steel target plate was used for acquisition of MALDI-TOF mass spectra (Bruker Daltonics, Billerica, MA). Matrices for MALDI-TOF MS were prepared by dissolving 2,5-dihydroxybenzoic acid $(20 \mathrm{mg} / \mathrm{mL})$ in a $7: 3$ $(\mathrm{v} / \mathrm{v})$ mixture of $0.1 \%(\mathrm{v} / \mathrm{v})$ trifluoroacetic acid and acetonitrile, and MS was performed with a Bruker Autoflex II MALDI-TOF/TOF mass spectrometer operated in the reflectron mode. Mass spectral data were analyzed using Bruker flexAnalysis software (version 3.0). Vesicles were extruded with a Lipex extruder (Transferra Nanosciences, Burnaby, BC). Dynamic light scattering was performed using a Brookhaven Instruments Corp. (Holtsville, NY) model 90Plus particle sizer. Circular dichroism (CD) spectra were acquired with an Applied Photophysics (Beverly, MA) Chirascan CD spectrometer.

ICMTs 4-[2,5-dioxo-2H-pyrrol-1(5H)-yl]-N,N,N-trimethylbutan-1-aminium iodide (light probe) and 4-[2,5-dioxo- $2 \mathrm{H}$ pyrrol-1 $(5 H)$-yl]-N,N,N-trimethyl- $d_{9}$-butan-1-aminium iodide (heavy probe) were synthesized as previously described. ${ }^{13}$

Construction of Multiple-Cysteine ChoA Mutants. Primers were designed to incorporate each mutation into template plasmid pCO117 (a PKK223-3 derivative) containing the wild-type Streptomyces ChoA gene ${ }^{27}$ via site-directed mutagenesis PCR (Table S1). Methylated template DNA was digested with $D p n \mathrm{I}$ for $1.5 \mathrm{~h}$ at $37^{\circ} \mathrm{C}$ and then inactivated by being heated for $20 \mathrm{~min}$ at $80{ }^{\circ} \mathrm{C}$. The remaining PCR products were transformed into $\mathrm{XL} 1$ Blue cells and grown overnight at $37{ }^{\circ} \mathrm{C}$ on Luria Broth (LB)-agar $(200 \mu \mathrm{g} / \mathrm{mL}$ ampicillin) plates. A single colony was picked and grown in 10 $\mathrm{mL}$ of LB medium $(200 \mu \mathrm{g} / \mathrm{mL}$ ampicillin $)$ overnight at $37^{\circ} \mathrm{C}$. Plasmids were purified from the culture by means of a Qiagen QIAprep Plasmid Mini-Prep Kit. Purified plasmid DNA was sequenced to verify the presence of the desired mutation.

Construction of Single-Cysteine and Disulfide ChoA Mutants. Plasmid pCO270, containing the cysteine-free
ChoA mutant gene C56A/C282A/C445A/C452A ligated into the NdeI to HindIII fragment of pET20b, was used as the template for site-directed mutagenesis PCR. Primers were designed to incorporate each cysteine mutation into the C56A/C282A/C445A/C452A ChoA gene (Table S2). Plasmid preparation and DNA sequencing were performed in the manner described above.

Expression and Purification of Wild-Type and Mutant ChoA. Plasmids containing either wild-type or mutant ChoA genes were transformed into BL21(DE3)pLysS competent Escherichia coli cells and grown overnight at $37^{\circ} \mathrm{C}$ on LB-agar plates containing ampicillin $(200 \mu \mathrm{g} / \mathrm{mL})$. A single colony was picked and used to inoculate $10 \mathrm{~mL}$ of LB medium, which was grown overnight $(16 \mathrm{~h})$ at $37{ }^{\circ} \mathrm{C}$ and then added to $1 \mathrm{~L}$ of $2 \times Y T$ medium containing ampicillin $(200 \mu \mathrm{g} / \mathrm{mL})$. The culture $(1 \mathrm{~L})$ was grown at $37{ }^{\circ} \mathrm{C}$ until an $\mathrm{OD}_{600}$ of 0.8 was reached. After the culture was cooled on ice, isopropyl $\beta$-D-1thiogalactopyranoside was added to a final concentration of $100 \mu \mathrm{g} / \mathrm{mL}$. The culture was incubated for an additional $20 \mathrm{~h}$ at $18{ }^{\circ} \mathrm{C}$ before the cell pellet was harvested by centrifugation at $4000 \mathrm{~g}$ for $20 \mathrm{~min}$. The pellet was resuspended in $50 \mathrm{~mL}$ of $50 \mathrm{mM}$ sodium phosphate buffer $(\mathrm{pH} 7)$ and lysed twice with a cell disrupter at 27000 psi. The cell debris was removed by centrifugation at $135000 \mathrm{~g}$ for $60 \mathrm{~min}$. The resulting supernatant was precipitated with $1 \mathrm{M}\left(\mathrm{NH}_{4}\right)_{2} \mathrm{SO}_{4}$ and centrifuged at $4000 \mathrm{~g}$ for $20 \mathrm{~min}$. The pellet was discarded, and $\left(\mathrm{NH}_{4}\right)_{2} \mathrm{SO}_{4}$ was added to a final concentration of $2 \mathrm{M}$. Following centrifugation at $4000 \mathrm{~g}$ for $20 \mathrm{~min}$, the supernatant was discarded, and the pellet was resuspended in $30 \mathrm{~mL}$ of $50 \mathrm{mM}$ sodium phosphate buffer $(\mathrm{pH} 7)$ and dialyzed overnight with a $10 \mathrm{~K}$ molecular weight cutoff (MWCO) membrane against 50 $\mathrm{mM}$ sodium phosphate buffer $(\mathrm{pH} 7)$. The dialysate was loaded onto a Whatman DE-52 cellulose anion exchange column pre-equilibrated with $50 \mathrm{mM}$ sodium phosphate buffer $(\mathrm{pH} 7)$. After elution with $50 \mathrm{mM}$ sodium phosphate buffer ( $\mathrm{pH} 7)$, fractions that contained ChoA, as determined by sodium dodecyl sulfate-polyacrylamide gel electrophoresis, were pooled and concentrated by precipitation with $2.5 \mathrm{M}$ $\left(\mathrm{NH}_{4}\right)_{2} \mathrm{SO}_{4}$ at room temperature. The pellet was collected after centrifugation at $4000 \mathrm{~g}$ for $20 \mathrm{~min}$ and resuspended in 5 $\mathrm{mL}$ of $50 \mathrm{mM}$ sodium phosphate buffer $(\mathrm{pH} \mathrm{7})$, and the suspension was then loaded onto a butyl sepharose column pre-equilibrated with $50 \mathrm{mM}$ sodium phosphate $(\mathrm{pH} 7)$ and 1 $\mathrm{M}\left(\mathrm{NH}_{4}\right)_{2} \mathrm{SO}_{4}$. Pure ChoA was eluted from the column using a gradient from $100 \% 50 \mathrm{mM}$ sodium phosphate $(\mathrm{pH} 7)$ with 1 $\mathrm{M}\left(\mathrm{NH}_{4}\right)_{2} \mathrm{SO}_{4}$ to $100 \% 50 \mathrm{mM}$ sodium phosphate $(\mathrm{pH} 7)$ over 3 column volumes. Fractions containing ChoA were concentrated using a $30 \mathrm{~K}$ MWCO spin column to a final concentration of $0.5-2 \mathrm{mg} / \mathrm{mL}$. The total protein yield per liter of cell culture varied from 1 to $5 \mathrm{mg}$.

ChoA Activity Assay. For measurement of ChoA activity, ChoA (final concentration, $20 \mathrm{nM}$ ) was added to the standard assay buffer containing $0.025 \%(\mathrm{w} / \mathrm{v})$ Triton X-100 and $0.02 \%$ $(\mathrm{w} / \mathrm{v})$ bovine serum albumin in $50 \mathrm{mM}$ sodium phosphate $(\mathrm{pH} 7)$ preincubated with cholesterol that had been dissolved in 2-propanol for $10 \mathrm{~min}$. The final 2-propanol concentration in the reaction mixture was $1 \%$. The formation of cholest-4-en3-one was then monitored at $240 \mathrm{~nm}$ and $37{ }^{\circ} \mathrm{C}$.

Preparation of Lipid Vesicles. For 1,2-dimyristoyl-snglycero-3-phosphocholine (DMPC)/cholesterol vesicles, dry lipids $(41 \mathrm{mg})$ and cholesterol $(7.75 \mathrm{mg})$ were mixed in $\mathrm{CHCl}_{3}$ in a round-bottom flask. For dipalmitoylphosphatidylcholine (DOPC)/1,2-dioleoyl-sn-glycero-3-phospho-L-serine 
(DOPS)/cholesterol vesicles, $47 \mathrm{mg}$ of DOPC, $5 \mathrm{mg}$ of DOPS, and $7.75 \mathrm{mg}$ of cholesterol were mixed in $\mathrm{CHCl}_{3}$ in a roundbottom flask. The solvent was removed by rotary evaporation for $20 \mathrm{~min}$, and any remaining traces of the solvent were removed under vacuum for an additional $30 \mathrm{~min}$. The resulting lipid/cholesterol mixture was resuspended in $50 \mathrm{mM}$ sodium phosphate $(\mathrm{pH} \mathrm{7)}$ via sonication. Following five rounds of freezing and thawing, $\sim 100 \mathrm{~nm}$ unilamellar vesicles were formed by extrusion of the suspension through a 50 or $100 \mathrm{~nm}$ filter 10 times at 400 psi. The size of the vesicles was confirmed by dynamic light scattering.

ICMT Labeling of ChoA. A reaction mixture containing 50 $\mathrm{mM}$ HEPES ( $\mathrm{pH} 7$ ) with or without lipid vesicles $[3 \mathrm{mM}$ in 50 $\mathrm{mM}$ sodium phosphate buffer $(\mathrm{pH})$ ] was incubated for 10 min at $30{ }^{\circ} \mathrm{C}$. ChoA was then added to a final concentration of $5 \mu \mathrm{M}$, and the mixture was incubated for $1 \mathrm{~min}$ at $30^{\circ} \mathrm{C}$. A heavy probe (final concentration, $50 \mu \mathrm{M}$ ) was added, and the solution was gently vortexed. At $0.5-60 \mathrm{~min}$ intervals, an aliquot was removed and added to $50 \mathrm{mM}$ (final concentration, $5 \mathrm{mM}$ ) light probe. To solubilize the vesicles and unfold the protein, sodium deoxycholate [final concentration, $1 \%(\mathrm{v} / \mathrm{v})]$ was added to each aliquot, which was then incubated at $70{ }^{\circ} \mathrm{C}$ for $1 \mathrm{~h}$. Finally, $200 \mathrm{ng}$ of trypsin or chymotrypsin was added to each aliquot, and digestion was allowed to occur for $16-18 \mathrm{~h}$ at 37 or $25{ }^{\circ} \mathrm{C}$, respectively. Acidification with $3 \%$ trifluoroacetic acid stopped the digestion and precipitated the sodium deoxycholate, which was removed by centrifugation at $15000 \mathrm{~g}$ for $10 \mathrm{~min}$. The supernatant was mixed with an equal volume of 2,5-dihydroxybenzoic acid matrix to prepare the sample for MALDI-TOF MS. The resulting mixture $(1 \mu \mathrm{L})$ was spotted twice onto an MTP384 target plate and air-dried before mass spectra were recorded.

MALDI-TOF MS. The percentage of first-step heavy-probe labeling at each time point was determined by calculating the integrated area of the heavy- and light-probe-labeled peptides and determining the heavy labeled area as a percentage of the total labeled area:

$$
\begin{aligned}
& \text { percent heavy labeled }(\% \mathrm{H}) \\
& =\frac{\text { area }_{\text {heavy labeled }}}{\text { area }_{\text {heavy labeled }}+\text { area }_{\text {light labeled }}}
\end{aligned}
$$

The percentage of heavy-probe labeling at each time point was then plotted and fit to the following equation:

$$
Y=Y_{\max }\left(1-\mathrm{e}^{-k t}\right)
$$

where $Y$ is the $\% \mathrm{H}$ at each time point, $Y_{\max }$ is the maximum \% $\mathrm{H}, k$ is the observed rate constant, and $t$ is the time.

Statistical significance was determined with multiple $t$ tests performed in GraphPad Prism 7 software, using the HolmSidak method with an $\alpha$ of 0.05 to correct for multiple comparisons.

ICMT Confirmation of Disulfide Bond Formation. ChoA mutant 4CA/T168C containing the additional disulfide mutations W81C and A367C was incubated with a 100-fold molar excess of TCEP overnight at $4{ }^{\circ} \mathrm{C}$. Reduction of the disulfide was confirmed by an assay of the activity as described above. Then treated WCAC and untreated WCAC (each at 5 $\mu \mathrm{M}$ ) were incubated with the heavy or light ICMT probe (5 $\mathrm{mM}$ ) for $1 \mathrm{~h}$ at $25{ }^{\circ} \mathrm{C}$. Because TCEP does not contain any thiols, subsequent ICMT labeling can occur in the presence of TCEP. Sodium deoxycholate was added to a final concentration of $1 \%(\mathrm{v} / \mathrm{v})$, and the protein was incubated for $1 \mathrm{~h}$ at
$70{ }^{\circ} \mathrm{C}$ before the addition of $200 \mathrm{ng}$ of trypsin or chymotrypsin. After incubation for $8-16 \mathrm{~h}$ at 37 or $25{ }^{\circ} \mathrm{C}$, respectively, the sodium deoxycholate was precipitated by the addition of trifluoroacetic acid (final concentration, 5\%), and the precipitate was pelleted by centrifugation for $10 \mathrm{~min}$ at $18000 \mathrm{~g}$. The supernatant was mixed in a 1:1 ratio with MALDI-TOF matrix, and the mixture was spotted twice onto an MTP384 target plate for mass spectrum acquisition.

CD Spectroscopy and Thermal Denaturation. CD spectra of fully reduced and oxidized WCAC ChoA mutants were acquired from 190 to $260 \mathrm{~nm}$ at $25^{\circ} \mathrm{C}$. Three scans were performed and averaged. Thermal melting curves were acquired at $222 \mathrm{~nm}$ from 2 to $95{ }^{\circ} \mathrm{C}$ in $2{ }^{\circ} \mathrm{C}$ increments. The temperature was stabilized for $1 \mathrm{~min}$ at each increment before measurements.

\section{RESULTS}

Design of Multiple-Cysteine ChoA Mutants. Eight different ChoA mutants were constructed and expressed to map the solvent-accessible surface of ChoA bound to the lipid bilayer. Each mutant had at least one surface residue replaced with cysteine. Mutants 7 and 8 contained a subset of the same mutations present in mutants 3-6 to serve as labeling controls. Surface residues were selected for mutation by inspection of the crystal structure [Protein Data Bank (PDB) entry 1MXT] to identify amino acids that were sufficiently similar to cysteine that their mutation would result in only minimal disruption of the overall integrity of the protein.

Using cholesterol solubilized in detergent micelles as a substrate, we assayed the catalytic activity of each mutant and compared it to that of the wild type. All the mutants catalyzed the conversion of cholesterol to cholest-4-en-3-one at rates comparable to the rate for the wild-type enzyme (Table 1). The tryptophan emission spectra of the mutants were compared to the spectrum of the wild type to ensure that the mutants were properly folded. The $\lambda_{\max }$ values of tryptophan emission for the mutants were nearly identical to the wild-type value, demonstrating they were folded like the wild type (Table 1).

Table 1. Michaelis-Menten Constants and Tryptophan

\begin{tabular}{|c|c|c|c|c|}
\hline & $k_{\text {cat }}\left(\mathrm{s}^{-1}\right)$ & $\begin{array}{c}K_{\mathrm{m}} \\
(\mu \mathrm{M})\end{array}$ & $\begin{array}{l}k_{\text {cat }} / K_{\mathrm{m}} \\
\left(\mathrm{M}^{-1} \mathrm{~s}^{-1}\right)\end{array}$ & $\lambda_{\max } \operatorname{Trp}$ \\
\hline wild type & $67 \pm 6$ & $13 \pm 4$ & $5.1 \times 10^{6}$ & 329 \\
\hline mutant $1, \mathrm{~L} 80 \mathrm{C}$ & $89 \pm 7$ & $11 \pm 3$ & $8.4 \times 10^{6}$ & 330 \\
\hline mutant 2, L274C & $75 \pm 8$ & $17 \pm 6$ & $4.5 \times 10^{6}$ & 328 \\
\hline $\begin{array}{l}\text { mutant 3, A32C/S129C/ } \\
\text { T371C/A423C }\end{array}$ & $23 \pm 1$ & $11 \pm 2$ & $2.0 \times 10^{6}$ & 332 \\
\hline $\begin{array}{l}\text { mutant 4, S153C/A205C/ } \\
\text { S312C/T435C }\end{array}$ & $68 \pm 8$ & $10 \pm 4$ & $7.0 \times 10^{6}$ & 328 \\
\hline mutant 5, T168C/A276C & $46 \pm 4$ & $14 \pm 3$ & $3.4 \times 10^{6}$ & 328 \\
\hline $\begin{array}{l}\text { mutant 6, A184C/T239C/ } \\
\text { A407C/A465C }\end{array}$ & $60 \pm 6$ & $13 \pm 4$ & $4.6 \times 10^{6}$ & 330 \\
\hline $\begin{array}{l}\text { mutant 7, A32C/T168C/ } \\
\text { S312C/A465C }\end{array}$ & $30 \pm 2$ & $12 \pm 3$ & $2.4 \times 10^{6}$ & 328 \\
\hline $\begin{array}{l}\text { mutant } 8, \text { A184C/A301C/ } \\
\text { T394C }\end{array}$ & $57 \pm 6$ & $11 \pm 4$ & $5.0 \times 10^{6}$ & 329 \\
\hline
\end{tabular}
Fluorescence Emission Maxima for Wild-Type $\mathrm{ChoA}^{a}$ and ChoA Mutants ${ }^{b}$

T394C

${ }^{a}$ Wild-type ChoA contains four native cysteines. ${ }^{b}$ Measured by cholest-4-en-3-one formation at $240 \mathrm{~nm}$. Measurements were performed in triplicate and averaged. Errors are standard errors of the mean. ${ }^{c}$ Excitation at $280 \mathrm{~nm}$. 
ICMT Scanning of ChoA Containing Multiple Surface Cysteines. To identify the region of ChoA that interacts with the membrane, we labeled the ChoA mutants $(5 \mu \mathrm{M})$ with the ICMT heavy probe $(50 \mu \mathrm{M})$ in the presence or absence of 100 $\mathrm{nM}$ DMPC and cholesterol (3:1, $3 \mathrm{mM}$ DMPC) vesicles at 30 ${ }^{\circ} \mathrm{C}$ for $60 \mathrm{~min}$. Aliquots were removed at specified time points, and heavy labeling was quenched by the addition of an excess of the light probe $(50 \mathrm{mM})$. The heavy:light labeling ratio was then determined as a function of time by MALDI-TOF MS.

The labeling results were used to divide the residues into three categories: exposed, protected, and dynamic. Exposed residues were completely exposed to solvent and reached $100 \%$ labeling with the first-step probe in $<30$ s. Conversely, protected residues were not labeled with the first-step probe over a $60 \mathrm{~min}$ period. These residues reacted with the secondstep probe only after the protein had been unfolded. Finally, dynamic residues showed time-dependent first-step labeling over the course of the experiment, and thus, the data could be fit to the equation in Materials and Methods, and the $k_{\text {obs }}$ ICMT could be obtained.

Of the 13 residues tested by means of ICMT labeling, only L80C, T168C, and A205C exhibited dynamic labeling (Figure 2 ). These three residues also exhibited slightly slower rates of

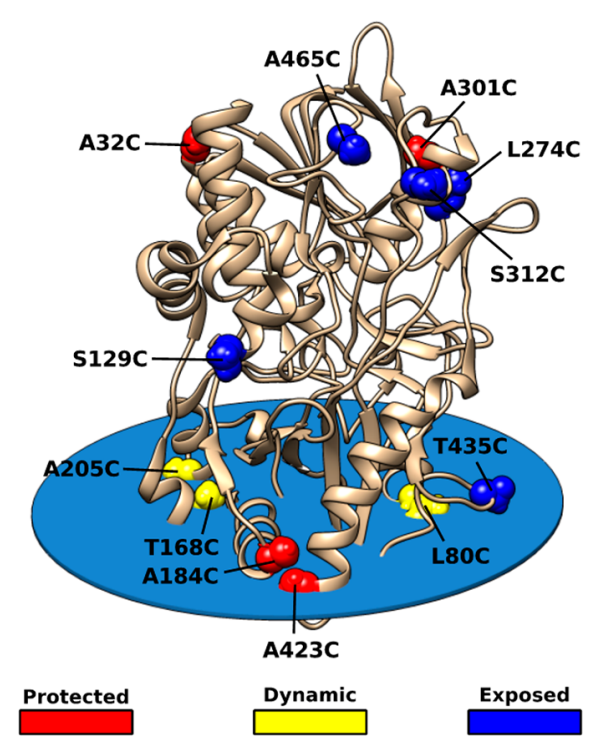

Figure 2. Summary of multiple-cysteine ICMT labeling experiments. Proposed orientation of ChoA (PDB entry $1 \mathrm{MXT}$ ) on the membrane, based on ICMT labeling of ChoA mutants containing multiple surface cysteines. Residues that exhibited protected, dynamic, and exposed labeling are colored red, yellow, and blue, respectively. Residue T371C (out of view beneath the plane) exhibited protected labeling.

labeling upon the addition of vesicles. The 10 remaining residues showed either protected or exposed labeling profiles that did not change upon addition of vesicles.

Quantification of ICMT Labeling Rates in ChoA Mutants Containing a Single Cysteine. To accurately quantify the rates of ICMT labeling, we constructed ChoA mutants that contained a single cysteine residue. The four native cysteines in $\mathrm{ChoA}$ were mutated to alanine, and separate L80C, A205C, and T168C mutations were constructed in the cysteine-free ChoA. These residues were chosen because they had been labeled more slowly upon the addition of vesicles in the experiments with ChoA containing multiple cysteines and thus were likely positioned at the protein-lipid interface.
Additional mutations (L369C and V176C) were constructed at residues chosen on the basis of their location relative to these three residues. The catalytic activity and tryptophan emission of each of these five single-cysteine ChoA mutants were assayed and determined to be comparable to those of the wild type (Table 2).

Table 2. Michaelis-Menten Constants and Tryptophan Fluorescence Emission Maxima for Wild-Type ChoA and Single-Cysteine ChoA Mutants ${ }^{a}$

$\begin{array}{lcccc} & k_{\text {cat }}\left(\mathrm{s}^{-1}\right) & K_{\mathrm{m}}(\mu \mathrm{M}) & k_{\text {cat }} / K_{\mathrm{m}}\left(\mathrm{M}^{-1} \mathrm{~s}^{-1}\right) & \lambda_{\max } \operatorname{Trp}(\mathrm{nm})^{b} \\ \text { wild type } & 20 \pm 9 & 11 \pm 1 & 1.8 \times 10^{6} & 329 \\ \text { L80C } & 22 \pm 6 & 12 \pm 2 & 1.8 \times 10^{6} & 328 \\ \text { A205C } & 14 \pm 9 & 8 \pm 2 & 1.1 \times 10^{6} & 328 \\ \text { T168C } & 23 \pm 7 & 14 \pm 0.3 & 1.6 \times 10^{6} & 327 \\ \text { L369C } & 21 \pm 5 & 14 \pm 3 & 1.4 \times 10^{6} & 328 \\ \text { V176C } & 38 \pm 7 & 19 \pm 3 & 2.0 \times 10^{6} & 328\end{array}$

${ }^{a}$ Measured by cholest-4-en-3-one formation at $240 \mathrm{~nm}$. Measurements were performed in triplicate and averaged. Errors are standard errors of the mean. ${ }^{b}$ Excitation at $280 \mathrm{~nm}$.

The single-cysteine ChoA mutants were labeled with ICMT probes under the conditions described above for the multiplecysteine mutants. Representative MALDI-TOF spectra for T168C ICMT labeling are shown with and without vesicles in panels A and B of Figure 3, respectively, at 30 s, 10 min, and 60 min, and the progress of ICMT labeling over time for residue $\mathrm{T} 168 \mathrm{C}$ is shown in Figure 3C. The data were fit to a one-phase exponential association equation to obtain $k_{\mathrm{obs}}$ ICMT for each labeling condition. The $k_{\mathrm{obs}}^{\text {ICMT }}$ values for all of the singlecysteine mutants are listed in Table 3 and shown graphically in Figure 3D. Time-course plots for the other four single-cysteine mutants are shown in Figure S1.

In the absence of vesicles, A205C was labeled approximately 20 times faster than $\mathrm{T} 168 \mathrm{C}$ was. However, the overall change in ICMT accessibility upon addition of vesicles was greater for T168C: in the presence of vesicles, T168C was labeled at 1 / 3 rd of the rate that it was labeled in buffer alone, whereas A205C was labeled at half of the rate. In contrast, mutant L80C showed no difference in labeling rate upon addition of vesicles.

To establish that these changes in accessibility were due to global protein interactions with the membrane, we constructed additional single-cysteine mutants in the same region as T168C and A205C, but on two different individual protein loops. Mutant L369C exhibited a labeling profile similar to that of A205C; the labeling rate in the presence of vesicles was half of that in buffer alone. In contrast, mutant V176C exhibited rapid labeling in the absence of vesicles; the labeling reaction was complete within $30 \mathrm{~s}$. Because labeling was so fast, the data could not be fit accurately enough to determine $k_{\text {obs }}^{\text {ICMT }}$, and we estimated the rate to be at least $35 \mathrm{~min}^{-1}$. Upon addition of vesicles, the rate of $\mathrm{V} 176 \mathrm{C}$ labeling was less than $1 / 300$ th of that in the absence of vesicles.

To determine the effects of vesicle size and electrostatic interactions, mutant $\mathrm{T} 168 \mathrm{C}$ was labeled in the presence of 150 $\mathrm{nm}$ large unilamellar vesicles (LUVs) and $50 \mathrm{~nm}$ small unilamellar vesicles (SUVs) with 10\% anionic lipid DOPS. Labeling was also performed in the presence of $10 \%$ DOPS LUVs with 10 and $100 \mathrm{mM} \mathrm{NaCl}$. No significant differences in labeling were observed (Figure S4). Vesicles that contained no cholesterol also did not affect labeling rates (data not shown). 

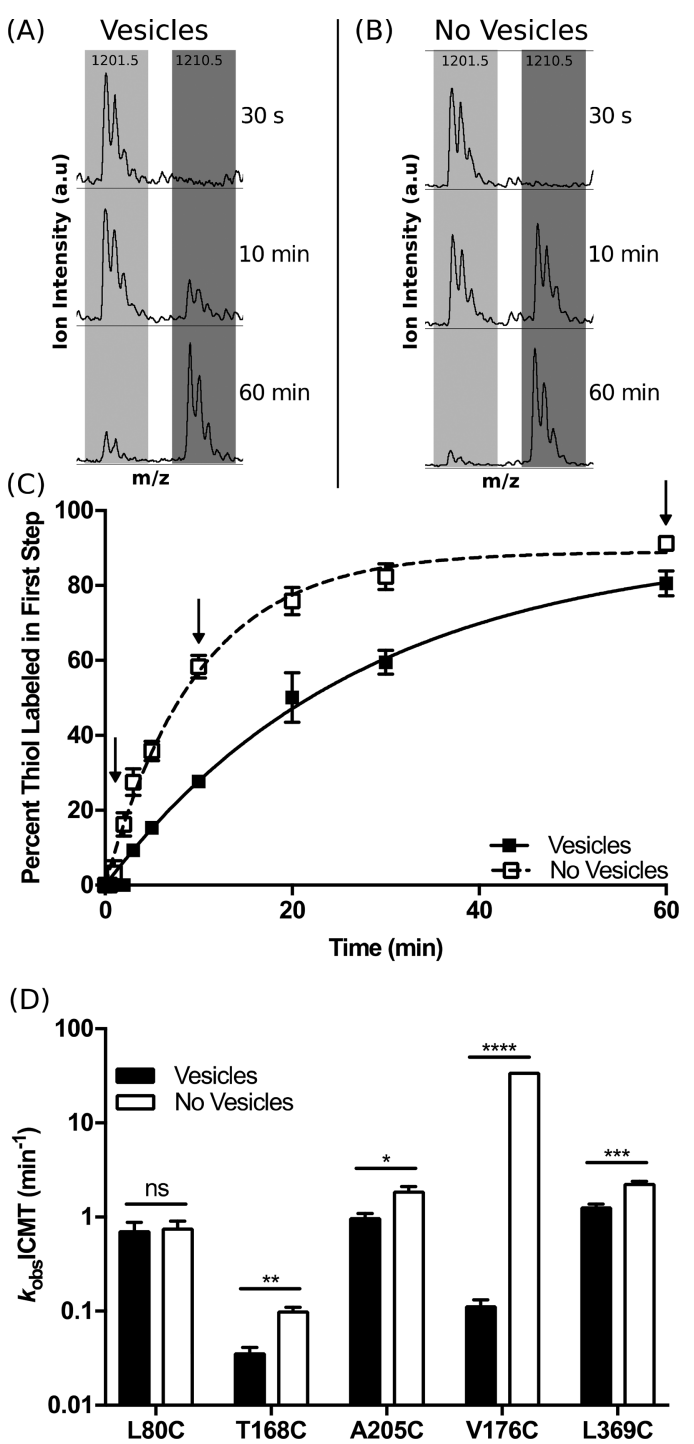

Figure 3. ICMT labeling analysis and rates for T168C and additional single-cysteine ChoA mutants. MALDI-TOF spectra of the singlecysteine T168C mutant at $30 \mathrm{~s}, 10 \mathrm{~min}$, and $60 \mathrm{~min}$ (A) with 3:1 $\mathrm{DMPC} /$ cholesterol (3 mM DMPC) vesicles and (B) without vesicles. Peaks corresponding to light-labeled peptides are highlighted in light gray. Peptides labeled with the heavy probe are highlighted in dark gray. (C) Temporal dependence of the ICMT labeling of T168C. Curves are fit to the one-phase exponential association equation to obtain $k_{\mathrm{obs}}^{\text {ICMT }}$ in the presence and absence of vesicles. Arrows indicate time points represented in panels A and B. Labeling data for all five single-cysteine mutants were analyzed in the same manner. (D) Values of $k_{\text {obs }}^{\text {ICMT }}$ for all of the single-cysteine ChoA mutants in the presence and absence of vesicles. Error bars are standard errors of the mean. ns, not significant. ${ }^{*} p<0.05 . * * p<0.01$. $* * * p<0.001$. $* * * * p<0.0001$.

Effect of a Disulfide Bond at the Protein-Lipid Interface. Engineering de novo disulfide bonds can limit conformational changes and alter substrate access channels. $^{28,29}$ In this study, we limited the mobility of helix $72-$ 86 by introducing a disulfide bond between residue W81 (on helix 72-86) and residue A367, which is on an adjacent flexible loop (residues 364-372) (Figure 4). The residues to be incorporated into the disulfide bond were selected on the basis of thermodynamic calculations performed with the Disulfide by Design program. ${ }^{30}$ The average energy required
Table 3. Observed Rates of First-Step ICMT Labeling of Single-Cysteine ChoA Mutants

\begin{tabular}{|c|c|c|c|}
\hline & \multicolumn{2}{|c|}{$k_{\mathrm{obs}}^{\text {ICMT }}\left(\mathrm{min}^{-1}\right)$} & \multirow{2}{*}{ 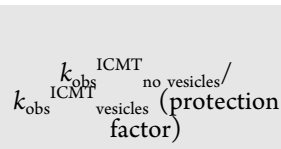 } \\
\hline & vesicles $^{a}$ & no vesicles & \\
\hline L80C & $0.72 \pm 0.15$ & $0.78 \pm 0.18$ & 1 \\
\hline $\mathrm{T} 168 \mathrm{C}$ & $0.036 \pm 0.0042$ & $0.10 \pm 0.0073$ & 3 \\
\hline A205C & $1.00 \pm 0.086$ & $1.92 \pm 0.19$ & 2 \\
\hline V176C & $0.12 \pm 0.016$ & $35.00^{b}$ & 300 \\
\hline L369C & $1.3 \pm 0.080$ & $2.3 \pm 0.091$ & 2 \\
\hline
\end{tabular}

${ }^{a} \mathrm{DMPC} /$ cholesterol (3:1, $3 \mathrm{mM} \mathrm{DMPC)}$ vesicles. ${ }^{b}$ Equal to $100 \%$ first-step labeling in $30 \mathrm{~s}$.

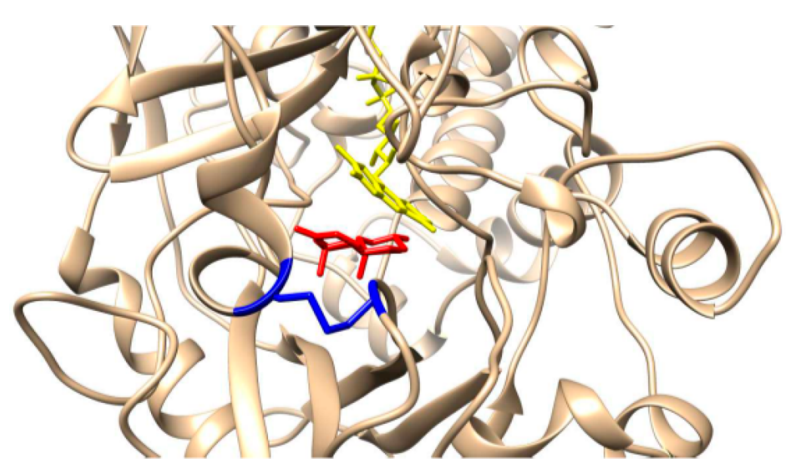

Figure 4. Position of the disulfide bond introduced in ChoA. In this depiction, ChoA (PDB entries 1MXT and 1COY) is viewed from the perspective of the lipid bilayer. Residue W81 on helix 72-86 and residue A367 on loop 364-372 were mutated to cysteine to enable formation of a disulfide bond (blue). The substrate analogue androsterone (red) is positioned in the active site. The flavin adenine dinucleotide cofactor is colored yellow.

to form a disulfide bond is $1.07 \mathrm{kcal} / \mathrm{mol},{ }^{31}$ and the energy to form a disulfide between $\mathrm{C} 81$ and $\mathrm{C} 367$ was calculated to be $1.05 \mathrm{kcal} / \mathrm{mol}$; therefore, we anticipated that these cysteines would readily form a disulfide bond. The ChoA disulfide mutant W81C-A367C (WCAC) also contained the T168C mutation, inclusion of which allowed us to use ICMT labeling to determine whether the disulfide bond affected membrane binding.

We confirmed the presence of the disulfide bond by ICMT labeling of both WCAC and WCAC treated with the reducing agent tris(2-carboxyethyl)phosphine (TCEP). The cysteines involved in the disulfide bond reacted with ICMT probes only after treatment with TCEP. Residue T168C, which was not expected to participate in the disulfide bond, was labeled as expected even in the absence of TCEP treatment (Figure S2A,B).

We evaluated the catalytic activity of WCAC as well as the activities of mutants containing only W81C or A367C (Table 4). Formation of the disulfide bond rendered the enzyme catalytically inactive. Treatment with a 100 -fold molar excess of TCEP restored the activity to wild-type levels. The activities of single-cysteine mutants W81C and A367C were comparable to the activity of wild-type ChoA.

Intrinsic tryptophan fluorescence $\lambda_{\max }$ of WCAC was slightly blue-shifted (by $5 \mathrm{~nm}$ ) from that of the wild type and remained unchanged upon treatment with TCEP. The $\lambda_{\max }$ values of W81C and A267C were also comparable to the value of the wild type. The secondary structures of the treated and untreated WCAC were compared by $\mathrm{CD}$ spectroscopy and 
Table 4. Characterization of the Disulfide ChoA Mutant W81C-A367C and Associated Control Mutants ${ }^{a}$

\begin{tabular}{|c|c|c|c|c|c|}
\hline & $k_{\text {cat }}\left(\mathrm{s}^{-1}\right)$ & $\begin{array}{c}K_{\mathrm{m}} \\
(\mu \mathrm{M})\end{array}$ & $\underset{\left(\mathrm{M}^{-1} \mathrm{~s}^{-1}\right)}{k_{\mathrm{ct}} / K_{\mathrm{m}}}$ & $\begin{array}{l}\lambda_{\max } \\
\operatorname{Trp}_{(\mathrm{nm})}\end{array}$ & $\begin{array}{c}k_{\text {obs ICMT }}^{\text {ICMT vesicles }} / \\
k_{\text {obs vesicles }} \\
\text { (protection } \\
\text { factor) }\end{array}$ \\
\hline wild type & $20 \pm 9$ & $11 \pm 1$ & $1.8 \times 10^{6}$ & 329 & $\mathrm{na}^{c}$ \\
\hline $\begin{array}{l}\text { W81C- } \\
\text { A367C/ } \\
\text { T168C }\end{array}$ & 0.0 & $\mathrm{~nm}^{b}$ & $\mathrm{~nm}^{b}$ & 323 & 3 \\
\hline $\begin{array}{l}\text { W81C- } \\
\text { A367C/ } \\
\text { T168C } \\
\text { with } \\
\text { TCEP }\end{array}$ & $40 \pm 8$ & $19 \pm 4$ & $2.1 \times 10^{6}$ & 323 & 3 \\
\hline W81C & $21 \pm 5$ & $13 \pm 2$ & $1.6 \times 10^{6}$ & 326 & $\mathrm{nd}^{d}$ \\
\hline A367C & $29 \pm 2$ & $17 \pm 3$ & $1.7 \times 10^{6}$ & 328 & $\mathrm{nd}^{d}$ \\
\hline $\mathrm{T} 168 \mathrm{C}$ & & & & & 3 \\
\hline
\end{tabular}

${ }^{a}$ Measured by cholest-4-en-3-one formation at $240 \mathrm{~nm}$. Measurements were performed in triplicate and averaged. Errors are standard errors of the mean. ${ }^{b}$ Not measurable. ${ }^{c}$ Not applicable. ${ }^{d}$ Not determined.

found to be the same (Figure 5A). In addition, thermal denaturation curves of treated and untreated WCAC were acquired. The $T_{\mathrm{m}}$ of the nonreduced protein was $59.26{ }^{\circ} \mathrm{C}$, whereas that of the reduced protein was $47.76^{\circ} \mathrm{C}$ (Figure 5B). ChoA WCAC was labeled with ICMT reagents in the presence or absence of vesicles, in the manner described above. Under both conditions, the $k_{\text {obs }}{ }^{\text {ICMT }}$ of residue T168C in WCAC was nearly identical to that of single-cysteine T168C (Figure S3).

\section{DISCUSSION}

In this study, we mapped the protein-lipid interface of the interfacial enzyme ChoA. In a qualitative first step, we scanned the ChoA surface for interactions with cholesterol-containing vesicles. By using mutants that contained multiple surface cysteines, we localized the protein region that bound to the membrane. Then we targeted single-cysteine mutants to quantify labeling rates.

In multiple-cysteine labeling experiments, all but three cysteines displayed either fully protected or fully exposed labeling profiles under the two labeling conditions. Interpretation of these two extreme profiles is difficult, because it is possible that these residues are involved in conformational changes but nevertheless remain buried or exposed. However, the decreases in labeling rates in the presence of vesicles observed for L80C, T168C, and A205C indicate that these residues were oriented toward the membrane. These three residues are clustered near the substrate entrance channel of ChoA (Figure 2), a location that is consistent with the original structural hypothesis. ${ }^{32}$

The labeling rates for mutants L80C, A205C, and T168C and two additional proteins with mutations near the proteinlipid interface were quantified in ChoA containing a single cysteine (Table 3 and Figure 3D). The slower labeling rates of residues $\mathrm{A} 205 \mathrm{C}$ and $\mathrm{T} 168 \mathrm{C}$ upon addition of vesicles confirm that these residues made contact with the membrane surface. Additional residues V176C and L369C were also labeled more slowly in the presence of vesicles than in buffer alone. The fact that the labeling rates of $\mathrm{T} 168 \mathrm{C}$ and $\mathrm{V} 176 \mathrm{C}$ in the presence of vesicles were slower than the rates of $\mathrm{A} 205 \mathrm{C}$ and $\mathrm{L} 369 \mathrm{C}$ under the same conditions suggests that $\mathrm{T} 168 \mathrm{C}$ and $\mathrm{V} 176 \mathrm{C}$ were more deeply buried in the membrane. In buffer alone, residues A205C and L369C were labeled faster than T168C was, indicating that the two former residues were more solventexposed on the protein itself.

Labeling data for the L80C mutant suggest that this residue did not make contact with the membrane surface. However, L80C resides on helix $72-86$, which appears to be important for conformational changes that allow cholesterol to access the active site. ${ }^{3}$ Inspection of the crystal structure suggests that helix 72-86 flips to sit on top of the membrane surface. Thus, residue L80C was equally exposed to solvent in the presence and absence of vesicles. Evidence of the helix conformational change is supported by our data indicating that the catalytic activity of ChoA was completely inhibited when the movement of this helix was restricted by incorporation of a disulfide bond and that the reduction of the disulfide bond restored activity to wild-type levels (Table 4). Intrinsic tryptophan fluorescence spectra and CD spectra of oxidized and reduced ChoA WCAC indicate that the overall structure of the protein was not affected by the disulfide bond (Table 4 and Figure 3A). The ICMT labeling rates of residue $\mathrm{T} 168 \mathrm{C}$ in the WCAC mutant in the presence and absence of vesicles were comparable to the labeling rate of single-cysteine mutant T168C (Figure S3), indicating that the binding orientation of the protein toward the vesicles was unaffected by the disulfide bond. Thermal denaturation curves show that the disulfide bond increased the $T_{\mathrm{m}}$ of ChoA by $11.5^{\circ} \mathrm{C}$, demonstrating that the disulfide bond stabilized the protein (Figure 3B). These experiments confirm the involvement of the active site helix in substrate turnover.

Taken together, the quantitative ICMT experiments show that four residues (T168C, V176C, A205C, and L369C)
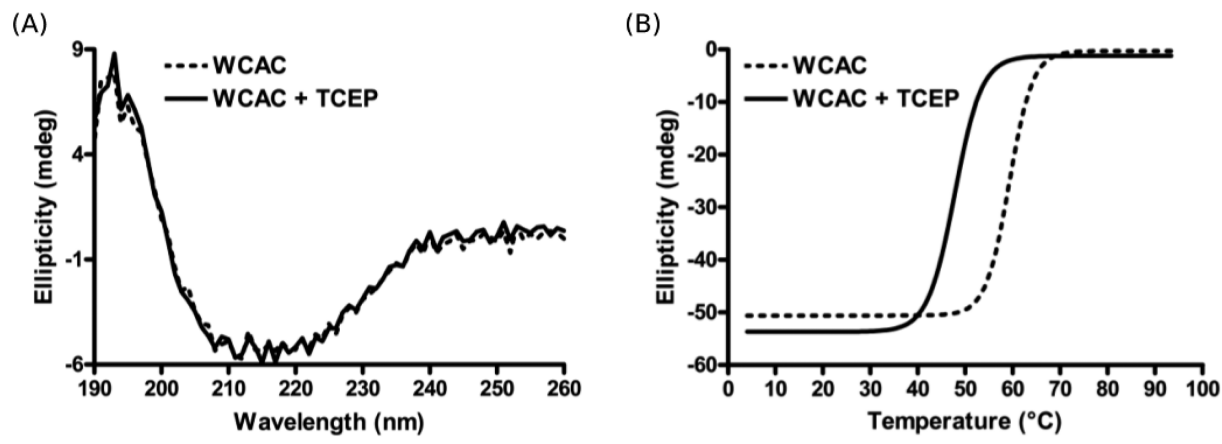

Figure 5. CD spectra and thermal denaturation curves for WCAC and WCAC treated with TCEP. (A) CD spectra of WCAC and WCAC with TCEP. (B) Thermal denaturation curves for WCAC and WCAC with TCEP. Data were fit to the Boltzmann sigmoid function, and $T_{\mathrm{m}}$ was calculated on the basis of the inflection point of each curve. 
engage with the membrane. These residues form a plane where the protein sits on top of the membrane (Figure 6). The depth

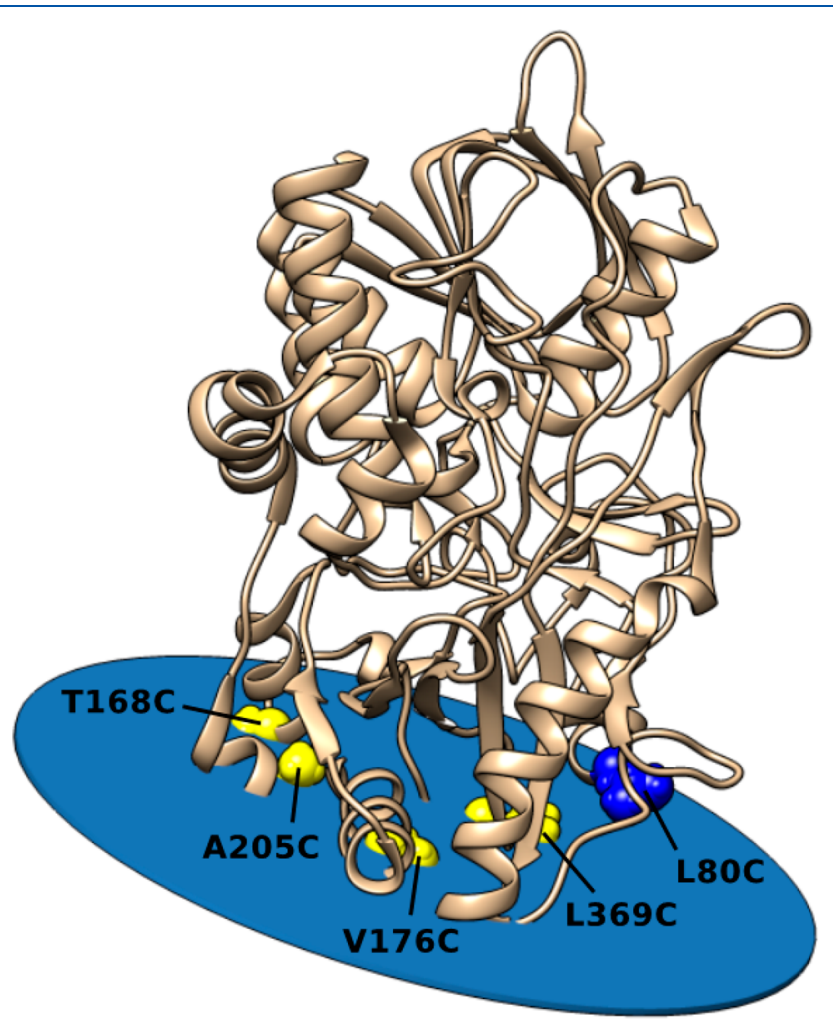

Figure 6. Refined orientation of ChoA on lipid vesicles. Orientation of ChoA (PDB entry 1MXT) on lipid vesicles, as indicated by singlecysteine ICMT labeling. Residues colored yellow exhibited slower labeling with ICMT probes in the presence of 3:1 DMPC/cholesterol vesicles than in their absence. Residue L80C (blue) was labeled at the same rate under both conditions.

to which the protein inserts into the membrane remains unclear. However, previous studies have determined that it must not insert very deeply and that the majority of the enzyme is on the exofacial side of the membrane as it sits on the surface. ${ }^{32}$ The relatively subtle $2-3$-fold changes in ICMT labeling rates for residues A205C, L369C, and T168C support this hypothesis. Although V176C has a large protection factor (300), the ICMT labeling rate upon lipid binding was 3 times that of $\mathrm{T} 168 \mathrm{C}$, indicating $\mathrm{T} 168 \mathrm{C}$ is the most deeply buried of the four residues.

The majority of the interface identified by ICMT contains more hydrophobic residues than charged residues (Figure 7).

Thus, membrane binding is driven by hydrophobicity rather than by electrostatic interactions. We expect that the structure of the protein interface remains consistent regardless of the charge of the vesicles, as labeling rates were not affected by anionic phospholipids or the ionic strength of the buffer (Figure S4). Additionally, control ICMT experiments in vesicles without cholesterol suggest that the presence of cholesterol does not affect the protein-membrane interface. These results further support our contention that the binding of ChoA to the membrane is mediated primarily through the interaction of the residues at the interface with the hydrophobic core of the membrane.

The ICMT method has the potential to be useful for determining protein-protein and protein-lipid interfaces in a

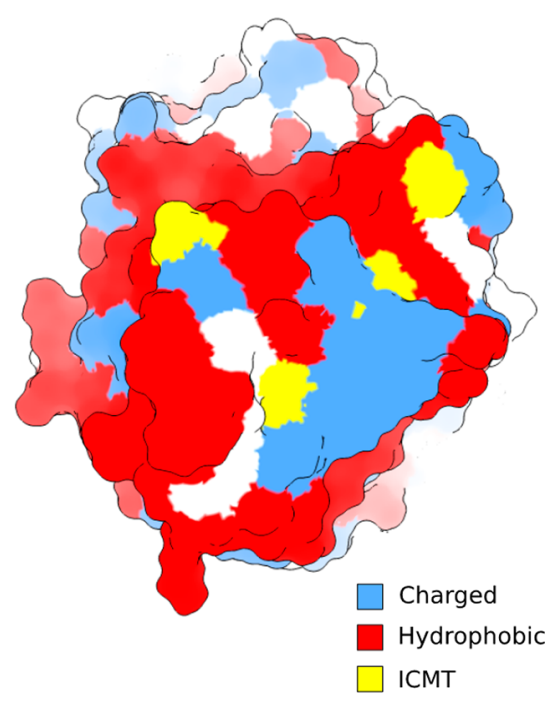

Figure 7. Amino acid content of the protein-lipid interface. Surface representation of ChoA from the perspective of the lipid bilayer. Hydrophobic, charged, and ICMT-identified residues are colored red, blue, and yellow, respectively (PDB entry $1 \mathrm{MXT}$ ).

wide variety of systems, including mammalian cell-based systems. Proteolysis and subsequent analysis by MALDITOF MS can be performed with heterogeneous samples. The limited number of steps involved reduces experimental error, and the short turnaround time lends itself to the type of kinetic analysis required for protein dynamics on millisecond to second time scales. The use of a charged mass tag enables ionization of the peptide of interest. Moreover, different proteases, such as chymotrypsin, can be used to ensure that hydrophobic interfacial or membrane proteins can be mapped. The method's reliance on the introduction of cysteine residues is ideal for membrane studies because cysteine reactivity is environment-dependent, and the energetic penalty for a cysteine to reside in a membrane environment is low. The use of a mass tag eliminates the loss of spatial resolution inherent to other methods involving target-probe conjugation. Thus, ICMT can be used to investigate difficult-to-detect protein-lipid interactions at the cell membrane, and we expect it can be adapted to investigate protein-protein interactions, as well.

\section{ASSOCIATED CONTENT}

\section{S Supporting Information}

The Supporting Information is available free of charge on the ACS Publications website at DOI: 10.1021/acs.biochem.8b00788.

Supplementary Tables S1 and S2 and Figures S1-S4 (PDF)

\section{AUTHOR INFORMATION}

\section{Corresponding Author}

*E-mail: nicole.sampson@stonybrook.edu.

ORCID

John E. Gadbery: 0000-0002-8998-2658

Nicole S. Sampson: 0000-0002-2835-7760

Funding

National Science Foundation Grant CHE1058439 (N.S.S.) and National Cancer Institute Grant R01CA166936 (N.S.S.). 


\section{Notes}

The authors declare no competing financial interest.

\section{ACKNOWLEDGMENTS}

Shearson Editorial Services (Cornwall, NY) provided Englishlanguage editing of the text of this paper.

\section{REFERENCES}

(1) Sampson, N. S., and Kwak, S. (2008) Catalysis at the Membrane Interface: Cholesterol Oxidase as a Case Study. Proceedings of $3 \mathrm{rd}$ International Symposium on Experimental Standard Conditions of Enzyme Characterizations (ESCEC), pp 13-22, Beilstein-Institut.

(2) Kreit, J., and Sampson, N. S. (2009) Cholesterol oxidase: physiological functions. FEBS J. 276, 6844-6856.

(3) Sampson, N. S., Kass, I. J., and Ghoshroy, K. B. (1998) Assessment of the role of an omega loop of cholesterol oxidase: a truncated loop mutant has altered substrate specificity. Biochemistry 37, 5770-5778.

(4) Pollegioni, L., Piubelli, L., and Molla, G. (2009) Cholesterol oxidase: biotechnological applications. FEBS J. 276, 6857-6870.

(5) Lario, P. I., Sampson, N., and Vrielink, A. (2003) Sub-atomic resolution crystal structure of cholesterol oxidase: what atomic resolution crystallography reveals about enzyme mechanism and the role of the FAD cofactor in redox activity. J. Mol. Biol. 326, 16351650.

(6) Zarychta, B., Lyubimov, A., Ahmed, M., Munshi, P., Guillot, B., Vrielink, A., and Jelsch, C. (2015) Cholesterol oxidase: ultrahighresolution crystal structure and multipolar atom model-based analysis. Acta Crystallogr., Sect. D: Biol. Crystallogr. 71, 954-968.

(7) Kass, I. J., and Sampson, N. S. (1998) Evaluation of the role of His447 in the reaction catalyzed by cholesterol oxidase. Biochemistry 37, 17990-18000.

(8) Kass, I. J., and Sampson, N. S. (1998) The importance of GLU361 position in the reaction catalyzed by cholesterol oxidase. Bioorg. Med. Chem. Lett. 8, 2663-2668.

(9) Li, J., Vrielink, A., Brick, P., and Blow, D. M. (1993) Crystal structure of cholesterol oxidase complexed with a steroid substrate: implications for flavin adenine dinucleotide dependent alcohol oxidases. Biochemistry 32, 11507-11515.

(10) Lyubimov, A. Y., Chen, L., Sampson, N. S., and Vrielink, A. (2009) A hydrogen-bonding network is important for oxidation and isomerization in the reaction catalyzed by cholesterol oxidase. Acta Crystallogr., Sect. D: Biol. Crystallogr. 65, 1222-1231.

(11) Yin, Y., Liu, P., Anderson, R. G., and Sampson, N. S. (2002) Construction of a catalytically inactive cholesterol oxidase mutant: investigation of the interplay between active site-residues glutamate 361 and histidine 447. Arch. Biochem. Biophys. 402, 235-242.

(12) Vrielink, A., and Ghisla, S. (2009) Cholesterol oxidase: biochemistry and structural features. FEBS J. 276, 6826-6843.

(13) Su, C. Y., London, E., and Sampson, N. S. (2013) Mapping peptide thiol accessibility in membranes using a quaternary ammonium isotope-coded mass tag (ICMT). Bioconjugate Chem. 24, $1235-1247$.

(14) Chakraborty, H., and Chattopadhyay, A. (2015) Excitements and challenges in GPCR oligomerization: molecular insight from FRET. ACS Chem. Neurosci. 6, 199-206.

(15) Ivanusic, D., Eschricht, M., and Denner, J. (2014) Investigation of membrane protein-protein interactions using correlative FRETPLA. BioTechniques 57, 188-191, 193-198.

(16) Kedziora, K. M., and Jalink, K. (2015) Fluorescence resonance energy transfer microscopy (FRET). Methods Mol. Biol. 1251, 67-82.

(17) Cafiso, D. S. (2014) Identifying and quantitating conformational exchange in membrane proteins using site-directed spin labeling. Acc. Chem. Res. 47, 3102-3109.

(18) Claxton, D. P., Kazmier, K., Mishra, S., and McHaourab, H. S. (2015) Navigating Membrane Protein Structure, Dynamics, and Energy Landscapes Using Spin Labeling and EPR Spectroscopy. Methods Enzymol. 564, 349-387.
(19) Fischer, A. W., Alexander, N. S., Woetzel, N., Karakas, M., Weiner, B. E., and Meiler, J. (2015) BCL::MP-fold: Membrane protein structure prediction guided by EPR restraints. Proteins: Struct., Funct., Genet. 83, 1947-1962.

(20) Malmberg, N. J., and Falke, J. J. (2005) Use of EPR power saturation to analyze the membrane-docking geometries of peripheral proteins: applications to C2 domains. Annu. Rev. Biophys. Biomol. Struct. 34, 71-90.

(21) Ladokhin, A. S. (1999) Analysis of protein and peptide penetration into membranes by depth-dependent fluorescence quenching: theoretical considerations. Biophys. J. 76, 946-955.

(22) Ladokhin, A. S. (2014) Measuring membrane penetration with depth-dependent fluorescence quenching: distribution analysis is coming of age. Biochim. Biophys. Acta, Biomembr. 1838, 2289-2295.

(23) Bednar, R. A. (1990) Reactivity and $\mathrm{pH}$ dependence of thiol conjugation to $\mathrm{N}$-ethylmaleimide: detection of a conformational change in chalcone isomerase. Biochemistry 29, 3684-3690.

(24) Karlin, A., and Akabas, M. H. (1998) Substituted-cysteine accessibility method. Methods Enzymol. 293, 123-145.

(25) Zhu, Q., and Casey, J. R. (2007) Topology of transmembrane proteins by scanning cysteine accessibility mutagenesis methodology. Methods 41, 439-450.

(26) Bogdanov, M. (2017) Mapping of Membrane Protein Topology by Substituted Cysteine Accessibility Method (SCAM). Methods Mol. Biol. 1615, 105-128.

(27) Molnar, I., Hayashi, N., Choi, K. P., Yamamoto, H., Yamashita, M., and Murooka, Y. (1993) Bacterial cholesterol oxidases are able to act as flavoprotein-linked ketosteroid monooxygenases that catalyse the hydroxylation of cholesterol to 4-cholesten-6-ol-3-one. Mol. Microbiol. 7, 419-428.

(28) Zhang, H., Kenaan, C., Hamdane, D., Hoa, G. H., and Hollenberg, P. F. (2009) Effect of conformational dynamics on substrate recognition and specificity as probed by the introduction of a de novo disulfide bond into cytochrome P450 2B1. J. Biol. Chem. $284,25678-25686$.

(29) Ma, K., Temiakov, D., Anikin, M., and McAllister, W. T. (2005) Probing conformational changes in T7 RNA polymerase during initiation and termination by using engineered disulfide linkages. Proc. Natl. Acad. Sci. U. S. A. 102, 17612-17617.

(30) Craig, D. B., and Dombkowski, A. A. (2013) Disulfide by Design 2.0: a web-based tool for disulfide engineering in proteins. BMC Bioinf. 14, 346.

(31) Dombkowski, A. A. (2003) Disulfide by Design: a computational method for the rational design of disulfide bonds in proteins. Bioinformatics 19, 1852-1853.

(32) Chen, X., Wolfgang, D. E., and Sampson, N. S. (2000) Use of the parallax-quench method to determine the position of the activesite loop of cholesterol oxidase in lipid bilayers. Biochemistry 39, 13383-13389. 